have greater benefits for women. Study strengths include the large nationally representative cohort followed from birth and prospective measures of CSEP, PF and covariates. However, the current examination based on a complete case analysis, disregards missing data, which will be addressed in future work.

\section{P02 DOES ALL HEALTH SERVICES AND DELIVERY RESEARCH (HSDR) GET PUBLISHED? A STUDY TO FOLLOW UP THE PUBLICATION STATUS OF COHORTS OF HSDR}

${ }^{1} \mathrm{AA}$ Ayorinde* ${ }^{2}{ }^{2}$ Williams, ${ }^{2} \mathrm{R}$ Mannion, ${ }^{3} \mathrm{~F}$ Song, ${ }^{4} \mathrm{M}$ Skrybant, ${ }^{1} \mathrm{SRJ}$ Lilford, ${ }^{1} \mathrm{YF}$ Chen ${ }^{1}$ Warwick Medical School, University of Warwick, Coventry, UK; ${ }^{2}$ Health Services Management Centre, University of Birmingham, Birmingham, UK; ${ }^{3}$ Norwich Medical School, University of East Anglia, Norwich, UK; ${ }^{4}$ Institute of Applied Health Research, University of Birmingham, Birmingham, UK

\subsection{6/jech-2019-SSMabstracts. 154}

Background Publication bias has been demonstrated in several high-profile cases associated with clinical trials of pharmaceuticals, but empirical evidence on this bias is scarce in HSDR, which concerns research to produce evidence on the quality, accessibility and organisation of health services. We set out to gauge the existence and scale of publication bias in quantitative HSDR by following up the publication status of four cohorts of studies identified from registries and conferences.

Methods We identified HSDR cohorts from four sources: (1) all quantitative studies funded by the NIHR HS\&DR Programme (and its predecessors) between 2007-2014 ( $n=100)$; (2) a random sample of quantitative HSDR studies registered with HSRProj (a US-based prospective registry of health service and public health research) and completed in 2012 $(n=100)$; (3) abstracts from the 2012 International Society for Quality in Health Care (ISQua) conference $(n=50)$ and (4) Health Services Research UK (HSRUK) conference, 2012-14 $(n=50)$. We checked the publication status of selected studies in 2018 by searching PubMed and Google. When no relevant publications were found, we contacted the investigators to verify the publication status. We compiled descriptive statistics for each cohort and used univariate analysis to explore the association between reporting of statistically significant findings and publication status.

Results Following online search of publications, we needed to contact investigators for $145 / 300(48 \%)$ of the studies and had a response rate of $60 \%(67 / 111)$ among those with valid contact details. Publication of findings in academic journals varies between cohorts: NIHR 64\%, HSRProj 75\%, ISQua $26 \%$ and HSRUK 70\%. When grey literature (including technical reports available online) was taken into account, the publication rates were NIHR 100\%, HSRProj 91\%, ISQua $32 \%$ and HSRUK $80 \%$. Statistically significant findings were reported by $79 \%(237 / 300)$ of the studies. There was no apparent association between reporting of statistically significant findings and publication in academic journals for the combined NIHR/HSRProj cohort (OR 1.19, 0.50-2.81) and conference cohort (OR 0.88, 0.25-3.10).

Discussion The rates of publication were generally high among HSDR studies that we sampled (which are likely to represent the high rectitude end of distribution), except in the ISQua cohort where the originators of the studies tended to be service providers rather than academic researchers. The power of our exploratory analysis was limited by the relatively small number of studies reporting non-significant findings and/or having a non-publication status. The publication policy of research funders (e.g. NIHR) appears to be effective in ensuring the publication of research findings.

\section{P03 A SYSTEMATIC REVIEW OF INTERVENTIONS IN COMMUNITY INFRASTRUCTURE (PLACES AND SPACES) TO BOOST SOCIAL RELATIONS AND COMMUNITY WELLBEING}

${ }^{1} \mathrm{AM}$ Bagnall ${ }^{*}$, 'J South, ${ }^{1} \mathrm{~K}$ Southby, ${ }^{1} \mathrm{~S}$ Di Martino, ${ }^{2} \mathrm{G}$ Pilkington, ${ }^{3} \mathrm{~B}$ Mitchell, ${ }^{2} \mathrm{~A}$ Pennington, ${ }^{2} \mathrm{R}$ Corcoran. ${ }^{1}$ School of Health and Community Studies, Leeds Beckett University, Leeds, UK; ${ }^{2}$ Institute of Psychology, Health and Society, University of Liverpool, Liverpool, UK; ${ }^{3}$ Leeds Business School, Leeds Beckett University, Leeds, UK

\subsection{6/jech-2019-SSMabstracts. 155}

Background Stakeholder engagement for the What Works Centre for Wellbeing's (WWCW) Community Wellbeing Evidence Programme identified 'boosting social relations' in communities as a priority policy-related topic. A scoping review of 34 reviews identified evidence gaps relating to social relations in the following areas: community infrastructure (places and spaces); interventions to reduce or prevent social isolation in adults $<60$ years; community engagement and volunteering; social network analyses. We developed 'community infrastructure (places and spaces)' as a systematic review, as this can be addressed at a local or regional level and has potential to produce immediate practical impact.

Methods We searched 11 bibliographic databases from 19972017, performed reference and citation checking, searched the websites of relevant organisations, and issued a call for evidence through the WWCW. We included studies which reported: interventions to improve or make alternative use of physical places and spaces at community or neighbourhood level; outcomes of social relations, community wellbeing and related concepts; quantitative, qualitative and mixed methods studies, and process evaluations. Two reviewers undertook study selection. One reviewer undertook data extraction and validity assessment, with a random $20 \%$ checked by a second reviewer. Validity of included studies was assessed using established checklists. Following thematic synthesis of qualitative data, a narrative synthesis was produced for each of eight intervention categories. The GRADE and CERQual approaches were used to rate the overall strength of evidence for each outcome.

Results 51 studies, mostly of poor to moderate quality, were included. The better quality evidence was qualitative, and most of the review's findings come from the thematic synthesis of qualitative evidence

There was moderate evidence that

i. Community hubs may promote social cohesion, increase social capital and build trust between people, widen social networks and increase interaction between people, and increase people's knowledge or skills; 